\title{
Associação da resistência à insulina em pacientes portadores de diabetes mellitus tipo 1 com o estado nutricional, perfil glicêmico e lipídico
}

\author{
Association of insulin resistance in patients with type 1 diabetes mellitus with nutritional \\ status, glycemic and lipid profile
}

\author{
Mariana Pimentel Gomes Souza ${ }^{1}$ \\ Orcid: https://orcid.org/0000-0002-5201-3394 \\ Maria Yasmin Paz Teixeira ${ }^{3}$ \\ Orcid: https://orcid.org/0000-0002-2054-62843 \\ Renan Magalhães Montenegro Júnior ${ }^{5}$ \\ Orcid: https://orcid.org/0000-0001-7287-8726
}

\author{
Lorena Taúsz Tavares Ramos ${ }^{2}$ \\ Orcid: https://orcid.org/0000-0002-2364-5499 \\ Nathalia Bernardo Marinho Leite ${ }^{4}$ \\ Orcid: https://orcid.org/0000-0001-5962-6997 \\ Synara Cavalcante Lopes $^{6}$ \\ Orcid: https://orcid.org/0000-0001-9879-1952
}

\begin{abstract}
Resumo
Introdução: A resistência à insulina, apesar de pouco investigada em indivíduos com Diabetes mellitus tipo 1, pode ser frequente nesse público. Assim, parâmetros acessíveis à prática clínica são necessários para facilitar o rastreio dessa condição. Objetivo: Avaliar a presença de resistência à insulina em pacientes com diabetes mellitus tipo 1 por meio do índice triglicerídeos/glicose e associar com estado nutricional, perfil glicêmico e lipídico. Métodos: Estudo original transversal realizado com 45 pacientes adultos ( $\geq 19$ anos) atendidos no ambulatório de Endocrinologia e Diabetes de um Hospital Universitário de Fortaleza-CE. Obtiveram-se, a partir dos prontuários, dados sociodemográficos, antropométricos (peso e altura), bioquímicos e clínicos (comorbidades e tratamento farmacológico) dos pacientes. Utilizaram-se os pontos de corte propostos para a população mexicana para classificação do índice triglicerídeos/glicose, considerando-se alterado, valores $\geq 4,55$ para mulheres e $\geq 4,68$ para homens. A normalidade das variáveis quantitativas foi avaliada por meio do teste de Shapiro Wilk e, para investigar associações utilizou-se o teste t de Student e o teste de MannWhitney. Adotou-se um nível de significância de 5\% para todos os testes utilizados. Resultados: Observou-se que 62,22\% dos indivíduos revelaram níveis alterados do índice triglicerídeos/glicose. Indivíduos que apresentaram níveis alterados revelaram valores mais elevados de glicemia de jejum, hemoglobina glicada, triglicerídeos, colesterol total e LDL-c $(p<0,05)$. Entretanto, não se observou associação significativa com HDL-c $(p=0,38)$, nem com Índice de Massa Corporal $(\mathrm{p}=0,14)$. Conclusões: $\mathrm{O}$ índice triglicerídeos/glicose associou-se significativamente com glicemia de jejum, hemoglobina glicada, triglicerídeos, colesterol total e LDL-c.
\end{abstract}

Palavras-chave: doenças do sistema endócrino; glicemia; distúrbios do metabolismo dos lipídeos; índice de massa corporal)

\footnotetext{
Abstract

${ }^{1}$ Universidade Federal do Ceará, Brasil. E-mail: mariana_pimentelgomes@hotmail.com

${ }^{2}$ Universidade Federal do Ceará, Brasil. E-mail: lo.tausz@gmail.com

${ }^{3}$ Universidade Federal do Ceará, Brasil. E-mail: yasminpazteixeira@gmail.com

${ }^{4}$ Universidade Federal de Pelotas, Brasil. E-mail: nathaliabermar@hotmail.com

${ }^{5}$ Universidade Federal do Ceará, Brasil. E-mail: renanmmjr@gmail.com

${ }^{6}$ Universidade Federal do Ceará, Brasil. E-mail: synaralopes@yahoo.com.br
}

Introduction: Insulin resistance, although little investigated in individuals with type 1 diabetes mellitus, may be frequent in this population. Thus, accessible parameters to clinical practice are necessary to facilitate the screening of this condition. Objective: To evaluate the presence 
of insulin resistance in patients with type 1 diabetes mellitus using the triglyceride / glucose index and associate it with nutritional status, glycemic and lipid profile. Methods: Original cross-sectional study carried out with 45 adult patients ( $\geq 19$ years old) treated at the Endocrinology and Diabetes outpatient clinic of a University Hospital in Fortaleza-CE. Sociodemographic, anthropometric (weight and height), biochemical and clinical data (comorbidities and pharmacological treatment) were obtained from the medical records. The cutoff points proposed for the Mexican population were used to classify the triglyceride / glucose index, considering altered, values $\geq 4.55$ for women and $\geq 4.68$ for men. The normality of the quantitative variables was assessed using the Shapiro Wilk test and, to investigate associations, the Student $t$ test and the Mann-Whitney test were used. A significance level of $5 \%$ was adopted for all tests used. Results: It was observed that $62.2 \%$ of the individuals showed altered levels of the triglycerides / glucose index. Individuals who had altered levels revealed higher values of fasting blood glucose, glycated hemoglobin, triglycerides, total cholesterol and LDL-c $(\mathrm{p}<0.05)$. However, there was no significant association with HDL-c $(p=0.38)$ or with Body Mass Index $(p=0.14)$. Conclusions: The triglyceride / glucose index as significantly associated with fasting glycemia, glycated hemoglobin, triglycerides, total cholesterol and LDL-c.

Keywords: endocrine system diseases; blood glucose; lipid metabolism disorders; body mass index.

\section{Introdução}

Resistência à insulina (RI) é definida como uma condição na qual a insulina não exerce sua função de forma efetiva, apesar da sua secreção endógena preservada ou reposição exógena. Dessa forma, quantidades de insulina maiores do que as consideradas normais são necessárias para exercer seu efeito. A RI pode ser influenciada pela etnia, puberdade, gestação, envelhecimento e comorbidades, como hipertensão, diabetes mellitus tipo 2 (DM2), obesidade e dislipidemia, doenças autoimunes, infecções e uso de medicações, como corticoides ${ }^{\mathbf{1 , 2}}$.

No Diabetes mellitus Tipo 1 (DM1), doença autoimune caracterizada pela destruição das células $\beta$-pancreáticas e ausência da produção de insulina, pouca atenção é dada à ocorrência de RI quando comparada ao DM2. No entanto, estudos já revelaram que, com a progressão da doença, esses indivíduos podem apresentar essa condição. Apesar disso, as explicações fisiopatológicas para essa associação ainda não foram totalmente elucidadas ${ }^{3}$.

A gênese da RI no DM1 é multifatorial e envolve fatores genéticos, ambientais e de estilo de vida. O histórico familiar de DM2 em pacientes com DM1 pode representar maior risco para 0 desenvolvimento de aspectos característicos de DM2, como a RI. Adicionalmente, aspectos relacionados ao estilo de vida, como sedentarismo e maus hábitos alimentares, podem contribuir para o desenvolvimento do sobrepeso e obesidade, pior controle glicêmico e necessidade aumentada de doses de insulina exógena, fatores que podem desencadear ou agravar a $\mathrm{RI}^{4}$.

O método considerado padrão ouro para a avaliação da sensibilidade à insulina é o método de clamp euglicêmico hiperinsulinêmico. No entanto, consiste em um método de alto custo, invasivo e não aplicável à prática clínica ${ }^{5}$. Dessa forma, $\mathrm{o}$ índice triglicerídeo glicose (TyG), o qual equivale ao produto entre glicemia e níveis séricos de triglicerídeos de uma mesma amostra sanguínea, representa uma alternativa para avaliar a RI de forma mais rápida, simplificada e com menor custo. Visto que, triglicerídeo e glicemia de jejum são parâmetros rotineiramente utilizados². Além disso, o TyG revelou prever melhor a RI do que o Homeostasis Model Assessment (HOMA-IR) quando comparados ao clamp euglicêmico hiperinsulinêmico, e correlacionou-se significativamente com parâmetros de adiposidade, metabólicos e marcadores de aterosclerose subclínica em indivíduos com DM2 e com tolerância normal à glicose 5 . 
Nesse contexto, considerando a necessidade de métodos mais acessíveis para avaliar RI na prática clínica e a escassez de estudos que investiguem a presença dessa condição em pacientes adultos com DM1, o presente estudo objetivou avaliar a presença de RI nesse público por meio do índice TyG e associar com estado nutricional, perfil glicêmico e lipídico.

\section{Materiais e Métodos}

\section{Amostra e tipo de estudo}

O presente estudo é do tipo transversal, retrospectivo com componente analítico e abordagem quantitativa, realizado com pacientes adultos com diagnóstico de DM1 atendidos no ambulatório de Endocrinologia e Diabetes de um Hospital Universitário, localizado na cidade de Fortaleza, Ceará.

O planejamento e execução do presente estudo atendeu aos princípios éticos recomendados pela Resolução 466 do Conselho Nacional de Saúde de 20126, tendo sido submetido e aprovado pelo Comitê de Ética em Pesquisa do hospital em questão sob CAAE $\mathrm{n}^{\circ}$ 21760719.5.0000.5045.

\section{Delineamento da pesquisa}

A seleção amostral incluiu pacientes atendidos durante o período de 2018 a 2020 , e a coleta de dados realizou-se entre os meses de agosto a outubro de 2020. Os dados utilizados foram colhidos a partir dos prontuários e formulários de atendimento nutricional previamente elaborados.

\section{Critérios de Inclusão e Exclusão}

Incluiu-se pacientes de ambos os sexos e com idade igual ou superior a 19 anos, visto que se pretendia obter uma visão geral do TyG, não especificando por sexo, e que a investigação de RI é mais coerente em indivíduos adultos e com mais tempo de doença (DM1). Definiu-se como critérios de exclusão: gestantes, pacientes com comprometimento da função renal e/ou hepática, ou que estavam em tratamento oncológico, condições que poderiam ter impacto sobre o estado metabólico dos indivíduos e interferir nos resultados de glicemia e triglicerídeos séricos, parâmetros utilizados para cálculo do TyG.

\section{Procedimentos}

Coletaram-se dados antropométricos de peso $(\mathrm{kg})$ e altura $(\mathrm{m})$ dos pacientes para avaliação do estado nutricional por meio do Índice de Massa Corporal (IMC: peso $(\mathrm{kg}) /$ altura $\left.(\mathrm{m})^{2}\right) \mathrm{de}$ acordo com a classificação da World Health Organization (WHO) ${ }^{7}$ para adultos e com a recomendação do Sistema de Vigilância Alimentar e Nutricional/Ministério da Saúde $^{8}$ para idosos ( $\geq 60$ anos). Em relação aos dados sociodemográficos e de estilo de vida, foram obtidas informações relativas ao sexo, idade, situação conjugal, escolaridade, renda, consumo de bebidas alcoólicas, tabagismo e prática de atividade física. Dados clínicos dos pacientes, como comorbidades associadas, tratamento farmacológico e dados bioquímicos também foram coletados.

$\mathrm{O}$ cálculo do índice TyG foi calculado por meio da seguinte fórmula: [Ln (triglicerídeos em jejum (mg / dL) x glicose em jejum (mg / dL)]/2, e utilizaramse os pontos de corte propostos para a população adulta mexicana, sendo valores de 4,55 para mulheres e de 4,68 para homens ${ }^{9}$. Visto que não existem pontos de corte definidos para a população brasileira. Dessa forma, considerou-se TyG alterado para os seguintes valores: $\geq 4,55$ para mulheres e $\geq 4,68$ para homens.

Os dados obtidos foram inseridos no programa Microsoft Office Excel ${ }^{\circledR}$ e em seguida foi utilizado o programa estatístico JAMOVI ${ }^{\circledR}$ e o Software $R \circledast$ para realização da análise estatística dos mesmos. Realizouse análise descritiva das variáveis em estudo, sendo as variáveis categóricas apresentadas em frequências simples e percentuais e as numéricas em forma de média (desvio-padrão) ou mediana (intervalo interquartil). Para avaliar a normalidade das variáveis quantitativas, foi utilizado o teste de Shapiro Wilk e, para 
investigar associações utilizou-se o teste $\mathrm{t}$ de Student e o teste de Mann-Whitney.
Adotou-se um nível de significância de 5\% para todos os testes utilizados.

\section{Resultados}

A amostra foi composta por 45 pacientes com DM1, sendo, predominantemente, do sexo feminino $(\mathrm{n}=30 ; 66,67 \%)$, com idade mediana de 37 (19-75) anos, renda de até 3 salários mínimos ( $\mathrm{n}=29 ; 64,44 \%)$ possuindo a partir do ensino médio completo $(\mathrm{n}=29 ; 64,44 \%)$ e sem companheiro (a) $(\mathrm{n}=14 ; 31,11 \%)$. Além disso, 86,67\% $(\mathrm{n}=39)$ da amostra negou etilismo e $97,78 \%(\mathrm{n}=44)$ negou tabagismo. O IMC mediano foi de $24 \mathrm{~kg} / \mathrm{m}^{2}(16,9-36,4)$ e $53,33 \%(\mathrm{n}=24)$ dos indivíduos não apresentavam sobrepeso, apesar da maioria negar prática de exercício físico $(n=30 ; 66,67 \%)$ (Tabela 1).

Tabela 1. Perfil nutricional, sociodemográfico e estilo de vida de pacientes com DM1. Fortaleza, 2020

\begin{tabular}{|c|c|c|}
\hline Variáveis & $\mathbf{N}$ & $\%$ \\
\hline \multicolumn{3}{|l|}{ Sexo } \\
\hline Masculino & 15 & 33,33 \\
\hline Feminino & 30 & 66,67 \\
\hline \multicolumn{3}{|l|}{ Renda } \\
\hline$\leq 3$ salários mínimos & 29 & 64,44 \\
\hline >3 salários mínimos & 6 & 13,33 \\
\hline Sem informação & 10 & 22,23 \\
\hline \multicolumn{3}{|l|}{ Escolaridade } \\
\hline$<$ Ensino médio completo & 8 & 17,78 \\
\hline$\geq$ Ensino médio completo & 29 & 64,44 \\
\hline Sem informação & 8 & 17,78 \\
\hline \multicolumn{3}{|l|}{ Situação conjugal } \\
\hline Com companheiro & 7 & 15,56 \\
\hline Sem companheiro & 14 & 31,11 \\
\hline Sem informação & 24 & 53,33 \\
\hline \multicolumn{3}{|l|}{ Etilismo } \\
\hline Sim & 6 & 13,33 \\
\hline Não & 39 & 86,67 \\
\hline \multicolumn{3}{|l|}{ Tabagismo } \\
\hline Fumante & 1 & 2,22 \\
\hline Não fumante & 44 & 97,78 \\
\hline \multicolumn{3}{|l|}{ Atividade física } \\
\hline Sim & 15 & 33,33 \\
\hline Não & 30 & 66,67 \\
\hline \multicolumn{3}{|l|}{ Estado nutricional* } \\
\hline Com sobrepeso & 19 & 42,22 \\
\hline Sem sobrepeso & 24 & 53,33 \\
\hline Sem informação & 2 & 4,45 \\
\hline Total & 45 & 100 \\
\hline
\end{tabular}

Legenda: *Sobrepeso em adultos (IMC $\geq 25 \mathrm{~kg} / \mathrm{m}^{2}$ ); excesso de peso em idosos (IMC $\geq 27 \mathrm{~kg} / \mathrm{m}^{2}$ ).

Fonte: Elaborada pelos autores.

Em relação às comorbidades, a mais relatada foi dislipidemia $(\mathrm{n}=10 ; 22,22 \%)$, seguida de hipertensão arterial sistêmica $(\mathrm{n}=6 ; 13,34 \%)$ e hipotireoidismo $\quad(\mathrm{n}=5$; 11,11\%). Quanto ao tratamento farmacológico, a maioria $(\mathrm{n}=32 ; 71,11 \%)$ fazia uso de insulinas análogas, mas somente $31,11 \% \quad(n=14)$ realizavam contagem de carboidrato. Além disso, dentre as medicações orais, as mais citadas foram as estatinas $(\mathrm{n}=12 ; 26,67 \%)$ (Tabela $2)$. 
Tabela 2. Perfil clínico de pacientes com DM1- comorbidades, tratamento farmacológico e nutricional. Fortaleza, 2020.

\begin{tabular}{|c|c|c|}
\hline & $\mathbf{N}$ & $\%$ \\
\hline \multicolumn{3}{|c|}{ Hipertensão Arterial Sistêmica } \\
\hline Sim & 6 & 13,34 \\
\hline Não & 39 & 86,66 \\
\hline \multicolumn{3}{|l|}{ Dislipidemia } \\
\hline Sim & 10 & 22,22 \\
\hline Não & 35 & 77,78 \\
\hline \multicolumn{3}{|c|}{ Doença Cardiovascular } \\
\hline Sim & 3 & 6,67 \\
\hline Não & 42 & 93,33 \\
\hline \multicolumn{3}{|l|}{ Hipertireoidismo } \\
\hline Sim & 1 & 2,22 \\
\hline Não & 44 & 97,78 \\
\hline \multicolumn{3}{|l|}{ Hipotireoidismo } \\
\hline Sim & 5 & 11,11 \\
\hline Não & 40 & 88,89 \\
\hline \multicolumn{3}{|c|}{ Antidiabéticos orais } \\
\hline Sim & 8 & 17,78 \\
\hline Não & 37 & 82,22 \\
\hline \multicolumn{3}{|l|}{ Tipo de insulina } \\
\hline Humanas & 2 & 4,44 \\
\hline Análogas & 32 & 71,11 \\
\hline Sem informação & 11 & 24,45 \\
\hline \multicolumn{3}{|l|}{ Estatina } \\
\hline Sim & 12 & 26,67 \\
\hline Não & 33 & 73,33 \\
\hline \multicolumn{3}{|c|}{ Anti-hipertensivos } \\
\hline Sim & 8 & 17,78 \\
\hline Não & 37 & 82,22 \\
\hline \multicolumn{3}{|c|}{ Contagem de CHO } \\
\hline Sim & 14 & 31,11 \\
\hline Não & 20 & 44,44 \\
\hline Sem informação & 11 & 24,45 \\
\hline Total & 45 & 100 \\
\hline
\end{tabular}

Legenda: CHO: carboidrato.

Fonte: Elaborada pelos autores.

Observou-se que $62,22 \%(\mathrm{n}=28)$ dos indivíduos revelaram níveis alterados de TyG. A média foi de $172 \mathrm{mg} / \mathrm{dL}( \pm 81,3)$ e
$115 \mathrm{mg} / \mathrm{dL}( \pm 126)$ para glicemia de jejum e trigliceridemia de jejum, respectivamente (Tabela 3).

Tabela 3. Índice TyG de pacientes com DM1. Fortaleza, 2020.

\begin{tabular}{|c|c|c|}
\hline Variável & $\mathbf{N}$ & $\%$ \\
\hline TyG & & \\
\hline Normal & 17 & 37,78 \\
\hline Alterado & 28 & 62,22 \\
\hline Total & 45 & 100 \\
\hline
\end{tabular}

Legenda: TyG: índice triglicerídeo glicose; TyG alterado para os seguintes valores: $\geq 4,55$ para mulheres e $\geq$ 4,68 para homens.

Fonte: Elaborada pelos autores. 
Em relação ao perfil glicêmico e lipídico, respectivamente, indivíduos que apresentaram níveis alterados de TyG revelaram valores mais elevados de glicemia de jejum (GJ), triglicerídeos (TG) e colesterol total $(\mathrm{CT})(\mathrm{p}<0,05)$. Entretanto, não se observou associação significativa com HDL-c (HDL-colesterol) $(\mathrm{p}=0,22)$, LDL-c (LDL-colesterol) $(p=0,07), \mathrm{HbA} 1 \mathrm{c}$ (hemoglobina glicada) $(\mathrm{p}=0,057)$ nem com $\operatorname{IMC}(\mathrm{p}=0,33)$ (Tabela 4).

Tabela 4. Perfil glicêmico e lipídico e IMC de pacientes com DM1 em relação ao índice TyG. Fortaleza, 2020.

\begin{tabular}{|c|c|c|c|c|c|}
\hline \multirow[b]{2}{*}{ Variável } & \multicolumn{2}{|c|}{ TyG alterado } & \multicolumn{2}{|c|}{ TyG normal } & \multirow[b]{2}{*}{$\mathbf{P}$} \\
\hline & $\mathbf{N}$ & Média (DP) & $\mathbf{N}$ & Média (DP) & \\
\hline Glicemia de jejum (mg/dL) & 28 & $216,6(63,7)$ & 17 & $98,4(45,9)$ & $<0,01 *$ \\
\hline £Hemoglobina glicada (\%) & 22 & $8,1(1,3)$ & 14 & $10,9(5,0)$ & $0,057^{*}$ \\
\hline Triglicerídeo (mg/dL) & 28 & $148,9(151,0)$ & 17 & $59,3(17,8)$ & $<\mathbf{0 , 0 1 *}$ \\
\hline £Colesterol Total (mg/dL) & 26 & $187,2(43,2)$ & 17 & $157,4(39,8)$ & $\mathbf{0 , 0 2} ¥$ \\
\hline £LDL-c (mg/dL) & 21 & $105(30,0)$ & 14 & $84,9(33,9)$ & $0,07 ¥$ \\
\hline £HDL-c (mg/dL) & 25 & $51,3(15,9)$ & 14 & $57,8(15,7)$ & $0,22 ¥$ \\
\hline fIMC $\left(\mathrm{kg} / \mathrm{m}^{2}\right)$ & 26 & $26,0(5,4)$ & 17 & $23,8(3,0)$ & $0,33 *$ \\
\hline
\end{tabular}

Legenda: p significativo quando <0,05; DP: desvio padrão; LDL-c: LDL-colesterol; HDL-c: HDL-colesterol; IMC: índice de massa corporal; ¥: teste t de Student; *: teste de Mann-Whitney; £: n não totaliza 45 por falta de informação no prontuário.

Fonte: Elaborada pelos autores.

\section{Discussão}

O presente estudo revelou presença de níveis alterados do índice TyG em $62,22 \%(\mathrm{n}=28)$ dos indivíduos com DM1, e este revelou associação significativa com GJ, TG e CT. No entanto, o índice TyG não apresentou associação significativa com HDL-c, LDL-c, HbA1c, nem com IMC.

Acredita-se que o desenvolvimento da RI em indivíduos com DM1 possa ser influenciado por fatores de estilo de vida, história familiar de DM2, alterações genéticas, e inclusive por hiperinsulinização derivada do próprio tratamento com insulina exógena ${ }^{3}$. No entanto, a hiperglicemia crônica também representa um importante contribuinte para RI, e o tratamento com insulina demonstrou otimizar o controle glicêmico e minimizar a $\mathrm{RI}^{\mathbf{1 0}}$. Além disso, a adiposidade, decorrente de inatividade física, dieta rica em açúcar e demais carboidratos de rápida absorção, é considerada um dos principais fatores desencadeantes de RI. Nesse sentido, a adiposidade pode exigir maiores doses de insulina por reduzir sua sensibilidade, ao mesmo tempo que maiores doses de insulina favorecem o ganho ponderal, o que motiva o desenvolvimento ou agrava a $\mathrm{RI}^{3}$.

Não somente o excesso de peso, mas especialmente a distribuição de gordura corporal apresenta papel central no desenvolvimento da RI. A gordura principalmente concentrada na região visceral é mais inflamatória, ou seja, apresenta maior capacidade de secretar citocinas pró-inflamatórias, como TNF- $\alpha$ e IL-6, as quais participam da gênese da RI por interferirem negativamente na ativação do receptor de insulina. Ademais, a gordura visceral é mais suscetível à lipólise, aumentando assim os níveis de ácidos graxos livres e o influxo destes no fígado e músculo esquelético, favorecendo, dessa forma, a RI nesses órgãos em decorrência da lipotoxicidade ${ }^{\mathbf{1 1}}$.

Nesse contexto, a RI hepática provoca aumento da gliconeogênese pelo fígado, levando à hiperglicemia e contribuindo com a produção excessiva de lipoproteína de densidade muito baixa (VLDL), elevando, consequentemente, os níveis séricos de $\mathrm{TG}^{\mathbf{1 1}}$. Apesar do presente 
estudo não ter avaliado a distribuição de gordura dos pacientes, isso pode justificar o fato da maioria dos pacientes terem revelado níveis alterados do índice TyG apesar do IMC mediano de $24,4 \mathrm{~kg} / \mathrm{m}^{2}$ e da ausência de associação significativa entre TyG alterado e IMC. Ou seja, embora classificados como eutróficos, existe a possibilidade de que esses indivíduos apresentassem elevada concentração de gordura abdominal independentemente do IMC, contribuindo assim para os resultados obtidos. Já em estudo epidemiológico com indivíduos com DM1, o IMC médio foi de $25,27 \mathrm{~kg} / \mathrm{m}^{2}$, em que $30,9 \%$ apresentaram sobrepeso e $13,5 \%$ obesidade ${ }^{\mathbf{1 2}}$. Enquanto que no estudo DCCT (Diabetes Control and Complications Trial) a média de IMC foi de $23,5 \mathrm{~kg} / \mathrm{m}^{213}$. Um estudo com adultos jovens saudáveis, com IMC médio de $24,2 \mathrm{~kg} / \mathrm{m}^{2}$, identificou RI através do TyG em 9,8\% dos homens e $17,4 \%$ das mulheres ${ }^{9}$. Não foi detectado na literatura nenhum estudo que utilizasse o TyG como marcador para RI em sujeitos com DM1.

Além do excesso de peso e concentração de gordura visceral, o padrão alimentar, aspecto não avaliado pelo presente estudo, também exerce importante influência na fisiopatologia de RI e síndrome metabólica (SM). Padrão alimentar baseado em consumo de bebidas açucaradas e refrigerantes diet, pães brancos, hambúrgueres e batata frita associou-se à RI e aumento do risco de DM2 em estudo com mais de 7.000 participantes adultos na Inglaterra ${ }^{\mathbf{1 4}}$. Estudo longitudinal de três anos de acompanhamento com adultos iranianos revelou redução significativa do risco de RI naqueles que seguiram um padrão alimentar baseado em plantas e com baixo teor de gordura $^{15}$. Em relação ao perfil lipídico, a adoção do padrão alimentar Mediterrâneo em pacientes adultos e idosos com alto risco cardiovascular minimizou a aterogenicidade de partículas de LDL-c e melhorou a funcionalidade de partículas de HDL-c em indivíduos com e sem diabetes ${ }^{\mathbf{1 6}}$. No entanto, estudos que abordem o impacto de padrões alimentares sobre a RI e demais componentes da síndrome metabólica em pacientes com DM1 são escassos na literatura.

A dislipidemia manifestada em pacientes com diabetes caracteriza-se frequentemente por hipertrigliceridemia, níveis elevados de LDL-c e reduzidos de HDL-c, condições que incitam o desenvolvimento de doença cardiovascular, a qual apresentou-se como principal causa de mortalidade em pacientes com DM1. Nesse contexto, a RI foi capaz de prever desfechos de doença arterial coronariana em pacientes com DM1 ${ }^{17}$. Estudo com brasileiros, incluindo indivíduos com DM2 e com tolerância normal à glicose, revelou correlação significativa entre o índice TyG e a distribuição e depósitos de gordura, parâmetros metabólicos, inclusive $\mathrm{CT}$, LDL-c, HDL-c, insulina de jejum, e marcadores de aterosclerose subclínica relacionados à $\mathrm{RI}^{5}$.

Estudo realizado com indivíduos não diabéticos demonstrou que o índice TyG, em comparação com outros marcadores, apresentou correlação superior com parâmetros bioquímicos associados à dislipidemia aterogênica e com $\mathrm{HbA} 1 \mathrm{c}^{4}$. Investigação realizada na região de Navarra na Espanha, com 5.014 pacientes sem DM1, evidenciou que níveis mais elevados do TyG não estiveram associados a importantes fatores de risco cardiovasculares, como HDL-C e LDL-C, sexo, idade, tabagismo, hipertensão e IMC. Porém, independentemente destes, o TyG esteve associado a um risco aumentado de doenças cardiovasculares, demonstrando ser um potente preditor de doença arterial coronária $^{18}$, afirmando a utilidade do índice na identificação de indivíduos em alto risco para um mau desfecho cardiovascular.

O termo diabetes duplo, o qual é descrito desde 1991, refere-se à condição onde há manifestação de características referentes ao DM1 e DM2, ou seja, ocorre a combinação de autoimunidade, deficiência de insulina e RI. Nesse sentido, destaca-se que a deficiência absoluta de insulina e a RI 
são fenômenos independentes e que a última pode ocorrer em indivíduos com ou sem diabetes ${ }^{19}$. Além disso, metanálise que avaliou RI em pacientes com DM1 através do clamp euglicêmico hiperinsulinêmico, revelou que a RI é uma manifestação importante em pacientes com DM1, envolvendo tecido hepático, periférico e adiposo, e que está presente tanto em sujeitos bem quanto mal controlados ${ }^{1}$. Estudo com mais de 31.000 pacientes adultos com DM1 identificou que 25,4\% destes também se encaixavam no critério diagnóstico para síndrome metabólica, proposto pelo Programa Nacional de Educação sobre Colesterol, o qual considera a circunferência da cintura, pressão arterial níveis de TG, HDL-c e GJ. Adicionalmente, evidenciou-se que pacientes com DM1 e $\mathrm{SM}$, denominado pelo autor como diabetes duplo, apresentaram prevalência significativamente maior de complicações macrovasculares e risco aumentado de complicações microvasculares (nefropatia e retinopatia) quando comparados aos indivíduos com DM1 sem SM"12.

Nesse cenário, levanta-se a possibilidade da RI ser uma das responsáveis pelo mau controle glicêmico de pacientes com DM1, apesar dos inúmeros avanços no tratamento insulínico e das tecnologias de monitorização glicêmica ${ }^{20}$. Metanálise envolvendo mais de 1.500 pacientes com DM1 revelou que o tratamento combinado de insulina com metformina durante 3 meses foi capaz de reduzir os níveis de glicemia de jejum e pósprandial, HbAlc e dosagem de insulina diária. Além de demonstrar benefícios sobre o perfil lipídico, minimizando os níveis de CT, LDL-c e colesterol não-HDL ${ }^{17}$.

Dentre as limitações do estudo, destaca-se o próprio delineamento transversal, que impossibilita análise de causa e efeito entre as variáveis. Além disso, considerando que um dos objetivos do estudo foi avaliar a associação do estado nutricional com o índice TyG, a utilização de parâmetros além do IMC seria conveniente, visto que a classificação do estado nutricional definida somente por meio desse índice torna-se falha $\mathrm{e}$ superficial, uma vez que o mesmo não avalia a composição corporal. A ausência de outros parâmetros para avaliar o estado nutricional e a não obtenção de alguns dados sociodemográficos e bioquímicos justifica-se pelo fato da pesquisa ter sido realizada a partir de dados secundários e durante a pandemia de COVID-19, o que dificultou a obtenção de determinadas informações, configurando-se assim, outra limitação.

Entretanto, o presente estudo tornase relevante visto que se propôs a investigar e foi capaz de identificar níveis alterados do índice TyG, parâmetro utilizado para identificar RI, em pacientes adultos com DM1. Além disso, revelou-se associação significativa do índice com importantes parâmetros de perfil glicêmico (GJ) e lipídico (CT e TG). Nesse sentido, o estudo emite um alerta para a possibilidade da presença e da possível necessidade de tratamento da RI para melhor controle metabólico e prevenção de complicações micro e macrovasculares nesses indivíduos. Assim, considerando a carência de estudos que investiguem a presença de RI no contexto do DM1, especialmente utilizando-se desse índice, o estudo torna-se pertinente para a literatura científica. Além disso, o estudo sugere a possibilidade do uso de um marcador mais acessível de RI para a prática clínica, visto que se baseia em parâmetros bioquímicos corriqueiramente utilizados.

\section{Conclusão}

$\mathrm{O}$ índice TyG associou-se significativamente com GJ, TG e CT, em um grupo de pacientes adultos com DM1. Indivíduos que apresentaram níveis alterados de TyG revelaram valores mais elevados dos parâmetros citados acima.

Estudos adicionais são necessários para avaliar o potencial de uso do TyG como marcador para estimar a presença de RI em pacientes com DM1, e para identificar quais fatores estão associados à 
sua ocorrência. Dessa forma, o tratamento seria mais direcionado e auxiliaria no melhor manejo glicêmico e metabólico, minimizando assim, o risco de complicações e mortalidade nesse público.

\section{Referências}

1. Donga E, Dekkers OM, Corssmit EP, Romijn JA. Insulin resistance in patients with type 1 diabetes assessed by glucose clamp studies: systematic review and meta-analysis. Eur $J$ Endocrinol 2015;173:101-9.

2. Sociedade Brasileira de Diabetes. Diretrizes da sociedade brasileira de diabetes 20192020. São Paulo: Clannad; 2019.

3. Šimonienė D, Platūkiene A, Prakapienė E, Radzevičienė L, Veličkiene D. Insulin Resistance in Type 1 Diabetes Mellitus and Its Association with Patient's Micro- and Macrovascular Complications, Sex Hormones, and Other Clinical Data. Diabetes Ther 2020;11:161-74.

4. Khan SH, Sobia F, Niazi NK, Manzoor SM, Fazal N, Ahmad F. Metabolic clustering of risk factors: evaluation of Triglyceride-glucose index (TyG index) for evaluation of insulin resistance. Diabetol Metab Syndr 2018;10:74.

5. Vasques ACJ, Novaes FS, Oliveira MS, Souza, JRM, Yamanaka, A, Pareja, JC, et al. TyG index performs better than HOMA in a Brazilian population: A hyperglycemic clamp validated study. Diabetes Res Clin Pract 2011; 93:98-100.

6. Brasil. Resolução No 466 de 12 de dezembro de 2012. Diretrizes e normas regulamentadoras de pesquisas envolvendo seres humanos. Diário Oficial da União 2012.

7. World Health Organization. Obesity: preventing and managing the global epidemic. Geneva; 1998. (Report of a WHO consultation on obesity, 276).

8. Coordenação-Geral de Alimentação e Nutrição, Departamento de Atenção Básica, Secretaria de Atenção à Saúde, Ministério da Saúde. Orientações para a coleta e análise de dados antropométricos em serviços de saúde: norma técnica do sistema de vigilância alimentar e nutricional - SISVAN. Brasília: Ministério da Saúde, 2011. (Série G. Estatística e Informação em Saúde).

9. Guerrero-Romero F, Villalobos-Molina R, Jiménez-Flores JR, Simental-Mendia LE, Méndez-Cruz R, Murguía-Romero M, et al. Fasting Triglycerides and Glucose Index as a Diagnostic Test for Insulin Resistance in Young Adults. Arch Med Res 2016; 47: $382-$ 87.

10. Kaul K, Apostolopoulou M, Roden M. Insulin resistance in type 1 diabetes mellitus. Metab.: Clin. Exp 2015; 64: 1629-39.

11. Moreira RO, Vilar L, Godoy-Matos AF. Síndrome Metabólica: Relevância e Implicações Clínica. In: Vilar L. Endocrinologia clínica. Rio de Janeiro: Guanabara Koogan, 2016.

12. Merger SR, Kerner W, Stadler M, Zeyfang A, Jehle P, Müller-Korbsch M, et al. Prevalence and comorbidities of double diabetes. Diabetes Res Clin Pract 2016; 119:48-56.

13. Gautier JF, Beressi JP, Leblanc H, Vexiau P, Passa P. Are the implications of the Diabetes Control and Complications Trial (DCCT) feasible in daily clinical practice? Diabetes Metab. 1996;22:415-19.

14. McNaughton SA, Mishra GD, Brunner EJ. Dietary patterns, insulin resistance, and incidence of type 2 diabetes in the Whitehall II Study. Diabetes Care 2008; 31:134348. 
15. Doostvandi T, Bahadoran Z, Mozaffari-Khosravi H, Tahmasebinejad Z, Mirmiran P, Azizi F. The association of dietary patterns and the incidence of insulin resistance after a 3-year follow-up: Tehran Lipid and Glucose Study. Asia Pac J Clin Nutr 2017; 26:531-38.

16. Hernáez Á, Castañer O, Elosua R, Pintó X, Estruch R, Salas-Salvadó J, et al. Mediterranean Diet Improves High-Density Lipoprotein Function in HighCardiovascular-Risk Individuals: A Randomized Controlled Trial. Circulation 2017; 135:633-43.

17. Liu YS, Chen CN, Chen ZG, Peng Y, Lin XP, Xu LL. Vascular and metabolic effects of metformin added to insulin therapy in patients with type 1 diabetes: A systematic review and meta-analysis. Diabetes Metab Res Ver 2020; 36:3334.

18. Sánchez-Íñigo L, Navarro-González D, Fernández-Montero A, Pastrana-Delgado J, Martínez JA. The TyG index may predict the development of cardiovascular events. Eur J Clin Invest 2016; 46:189-97.

19. Teupe B, Bergis K. Epidemiological evidence for "double diabetes". Lancet 1991; 337:361-62.

20. Khawandanah J. Double or hybrid diabetes: A systematic review on disease prevalence, characteristics and risk factors. Nutr Diabetes 2019; 9:33.

\section{Como citar este artigo:}

Souza MPG, Ramos LTT, Teixeira MYP, Leite NBM, R Montenegro Júnior RM, Lopes SC. Associação da resistência à insulina em pacientes portadores de diabetes mellitus tipo 1 com o estado nutricional, perfil glicêmico e lipídico. Rev. Aten. Saúde. 2021; 19(70): 7-16. 